\title{
Specific NuRD components are required for fin regeneration in zebrafish
}

\author{
Catherine Pfefferli, Fritz Müller, Anna Jaźwińska* and Chantal Wicky*
}

\begin{abstract}
Background: Epimorphic regeneration of a missing appendage in fish and urodele amphibians involves the creation of a blastema, a heterogeneous pool of progenitor cells underneath the wound epidermis. Current evidence indicates that the blastema arises by dedifferentiation of stump tissues in the vicinity of the amputation. In response to tissue loss, silenced developmental programs are reactivated to form a near-perfect copy of the missing body part. However, the importance of chromatin regulation during epimorphic regeneration remains poorly understood.

Results: We found that specific components of the Nucleosome Remodeling and Deacetylase complex (NuRD) are required for fin regeneration in zebrafish. Transcripts of the chromatin remodeler chd4a/Mi-2, the histone deacetylase hdac1/HDAC1/2, the retinoblastoma-binding protein rbb4/RBBP4/7, and the metastasis-associated antigen mta2/MTA were specifically co-induced in the blastema during adult and embryonic fin regeneration, and these transcripts displayed a similar spatial and temporal expression patterns. In addition, chemical inhibition of Hdac1 and morpholino-mediated knockdown of chd4a, mta2, and rbb4 impaired regenerative outgrowth, resulting in reduction in blastema cell proliferation and in differentiation defects.

Conclusion: Altogether, our data suggest that specialized NuRD components are induced in the blastema during fin regeneration and are involved in blastema cell proliferation and redifferentiation of osteoblast precursor cells. These results provide in vivo evidence for the involvement of key epigenetic factors in the cellular reprogramming processes occurring during epimorphic regeneration in zebrafish.
\end{abstract}

Keywords: NuRD, Blastema, Fin, Regeneration, Zebrafish

\section{Background}

In contrast to mammals, some vertebrates such as urodeles and teleost fish benefit from exceptional regeneration mechanisms. Zebrafish are able to regenerate different organs after injury, including heart, fins, retina, liver, and spinal cord, and have become a powerful model organism for regenerative studies [1-5]. The caudal fin displays rapid and robust regeneration, and therefore provides a well-established system to study appendage regeneration in vertebrates $[4,6,7]$.

The caudal fin of zebrafish is constituted of 16 to 18 bony fin rays (lepidotrichia), covered by an epidermis, and interconnected by soft inter-ray mesenchymal tissue [6]. Each individual bony ray consists of two concave hemirays that enclose a mesenchymal compartment composed

\footnotetext{
* Correspondence: anna.jazwinska@unifr.ch; chantal.wicky@unifr.ch Department of Biology, University of Fribourg, Ch. du Musée 10, CH-1700 Fribourg, Switzerland
}

of blood vessels, nerves, pigment cells, fibroblasts, and osteoblasts.

Upon amputation, the caudal fin is fully restored after approximately 3 weeks. This type of regeneration, called epimorphic regeneration, involves the formation of a blastema, a population of proliferating progenitor cells that arise from dedifferentiation of mesenchymal cells in the stump $[4,8]$. Regeneration of the caudal fin proceeds through three main steps: 1) wound healing, 2) blastema formation, and 3) regenerative outgrowth, including differentiation and patterning. Upon fin amputation, epidermal cells rapidly migrate to protect the wound and form a wound epidermis. Mesenchymal tissues in the stump then become disorganized, and cells start to proliferate and migrate distally, forming a blastema after approximately 24 to 48 hours post-amputation (hpa). During regenerative outgrowth, the blastema progenitor cells are maintained at the distal margin, 
while their daughter cells progressively redifferentiate in the proximal part of the fin regenerate. During this later phase, the fin regenerate can be subdivided into several compartments with distinct cellular and molecular properties [9-11].

The exact origin of blastema cells still remains unresolved. Recently, genetic cell-fate tracing studies have shown that the blastema is composed of a heterogeneous population of cells with restricted lineage fate and different tissue origin [12-14]. Thus, regeneration is achieved without cellular transdifferentiation. However, genetic ablation studies of osteoblasts prior to amputation have revealed that new bones are able to regenerate from non-osteoblast cells, suggesting that other cell types are plastic, and can transdifferentiate into osteoblasts to promote bone regeneration [15].

Animals with robust regenerative capacities are characterized by their flexibility to change gene expression in response to amputation. This cellular plasticity allows temporal suppression of differentiation genes and reactivation of developmental signaling pathways, which are required for the reconstitution of lost tissues [11,16-28].

Regulation of the chromatin structure is an important epigenetic mechanism, which has a direct influence on many biological processes. The Nucleosome Remodeling and Deacetylase (NuRD) complex is a multi-subunit complex widely expressed and evolutionarily conserved in animals and plants [29]. This complex is able to couple two important enzymatic functions: an ATP-dependent nucleosome remodeling activity catalyzed by the chromodomain helicase DNA binding proteins $\mathrm{CHD} 3 / 4$, also called $\mathrm{Mi}-2 \alpha / \beta$, and a deacetylase activity executed by the histone deacetylases HDAC1/2 [30-33]. Additionally, the NuRD complex is also constituted of other noncatalytic subunits, including the methyl-CpG-binding domain proteins $\mathrm{MBD} 2 / 3$, the retinoblastoma-binding proteins RBBP7/4, and the metastasis-associated proteins MTA1/2/3 [34]. The composition of the NuRD complex can also be changed by the incorporation of unique subunits, raising the possibility of functional specialization for these distinct complexes [35].

The NuRD complex has been shown to play important developmental roles in cell fate determination [34]. In Caenorhabditis elegans, the Mi-2 homolog LET-418 is required for proper differentiation of the vulva [36] and for repression of germline-specific genes in somatic cells [37]. In Drosophila melanogaster, $\mathrm{dMi}-2$ is essential for embryogenesis and germ cell development [38]. Yoshida et al. [39] have demonstrated that in mammals, Mi- $2 \beta$ functions in self-renewal and lineage choice of hematopoietic stem cells [39]. In addition, embryonic stem cells deficient in $m b d 3$ can initiate differentiation, but are not able to commit to specific lineages [40].
In this study, we investigated the potential role of the $\mathrm{Mi}-2 / \mathrm{NuRD}$ complex during fin regeneration in zebrafish. The zebrafish genome encodes several orthologs for every member of the vertebrate NuRD complex. However, we found that only one of each is expressed during fin regeneration. The orthologs of the NuRD components chd4a/Mi-2, hdac1/HDAC1/2, rbb4/RBBP4/7, and mta2/ MTA are all induced in the distal blastema during regeneration of the adult and embryonic caudal fin, and display similar expression patterns. Additionally, inhibition of these genes impairs regenerative outgrowth. Our data suggest that putative NuRD components are induced in the blastema during fin regeneration, and are involved in the maintenance of blastema cell proliferation and in redifferentiation during the regenerative outgrowth phase.

\section{Results}

One of the three Mi-2 orthologs, chd $4 a$, is specifically expressed in the blastema during fin regeneration

Mi-2, which is the core ATPase of the NuRD complex, is essential for regeneration and neoblast differentiation in the planarian Schmidtea mediterranea [41]. We therefore investigated whether Mi-2 could also be involved in zebrafish fin regeneration. A BLAST search of the zebrafish genome database (National Center for Biotechnology Information) identified three genes, chd4a (Gene ID: 558344), chd4b (Gene ID: 560622), and chd3 (Gene ID: 568230), which encode polypeptides with high similarity to human $\mathrm{Mi}-2$ proteins, also called CHD4 (or Mi-2 $\beta$ ) and CHD3 (or Mi-2 $\alpha$ ). Sequence alignment revealed high similarity between the three zebrafish Mi-2 homologs, with the main functional domains being conserved (see Additional file 1: Figure S1). Chd4a and Chd4b share $82 \%$ identity, while Chd3 shares 66\% identity with Chd4a and Chd4b. Moreover, Chd4a contains an additional domain, the AP endonuclease family 2 domain (AP2Ec) (see Additional file 1: Figure S1), which is not present in other Mi-2 orthologs. This evolutionarily conserved domain is associated with DNA damage repair and maintenance of genome stability [42].

To determine whether these putative Mi-2 orthologs might play a role in fin regeneration, we first examined their expression profiles during this process. Quantitative real-time-PCR (qRT-PCR) identified significant upregulation of chd $4 a$ transcripts in regenerating fins at 3 days post-amputation (dpa) compared with amputated fins collected immediately after amputation (0 hpa) (Figure 1A). No upregulation was observed for the two other Mi-2 homologs, chd $4 b$ and chd3, in regenerating fins (Figure 1A). The temporal and spatial expression pattern of these genes was also analyzed by in situ hybridization (ISH) in regenerating adult caudal fins at $3 \mathrm{dpa}$. Consistent with the qRT-PCR data, only chd4a was induced in adult regenerating fins (Figure 1B; see Additional file 1: Figure S2). chd4a 

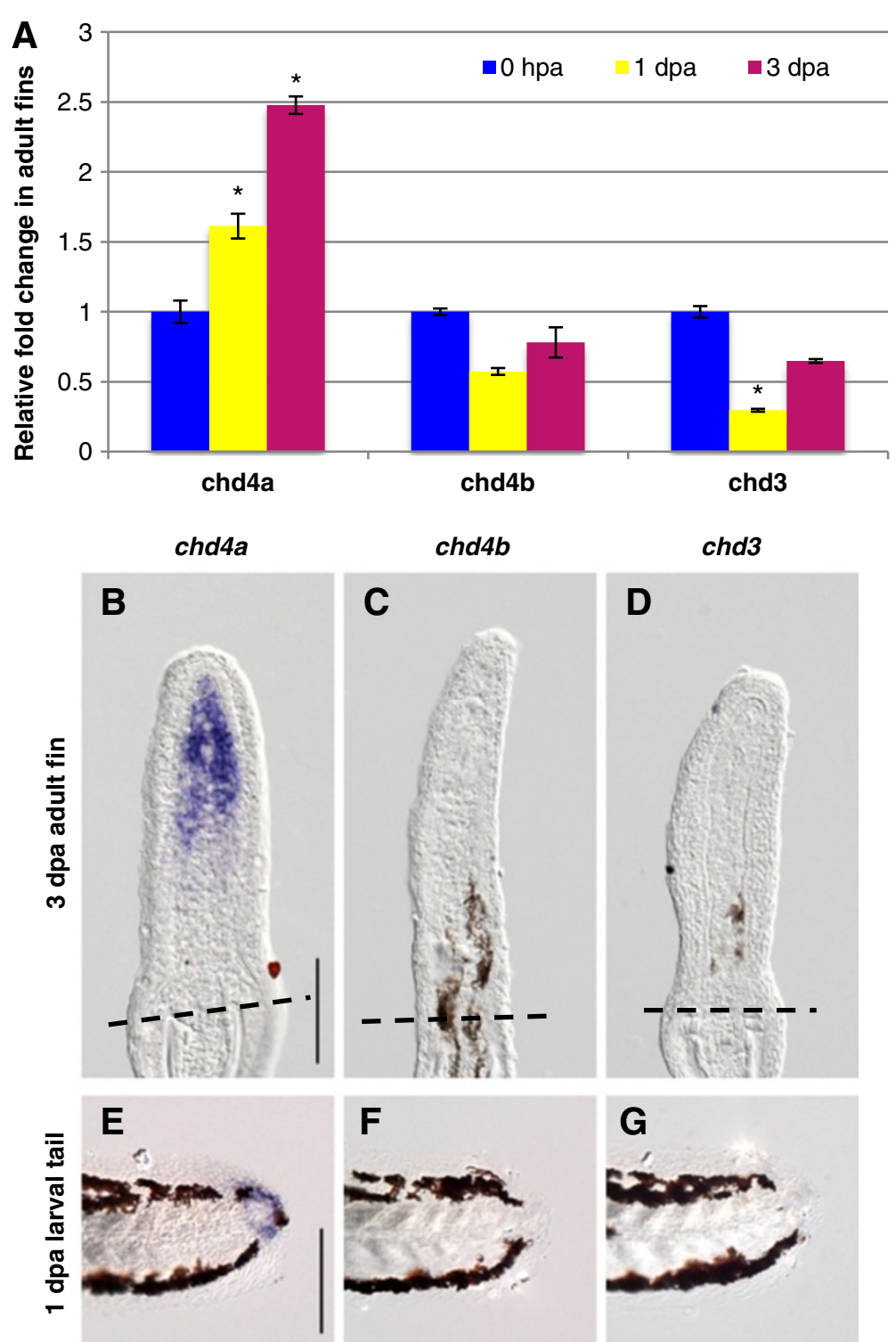

Figure 1 One of the three Mi-2 orthologs, chd4a, is specifically induced in the blastema during fin regeneration. (A) Quantitative real-timePCR analysis of $c h d 4 a$, chd4b, and chd3 mRNA in regenerating adult caudal fins at 1 day post-amputation (dpa) and 3 dpa relative to control fins at 0 hours post-amputation (hpa). Error bars represent the SEM. ${ }^{*} P<0.001$ (B-D) In situ hybridization with chd4a (B), chd4b (C), and chd3 (D) mRNA antisense probes on cryosections of regenerating adult caudal fins at $3 \mathrm{dpa}$. Dashed lines indicate the amputation plane. (E-G) Whole-mount in situ hybridization with chd4a (E), chd4b (F), and chd3 (G) antisense probes in 1 dpa larval tails. Scale bar: $100 \mu \mathrm{m}$.

transcripts were specifically located in the blastema, but not in the adjacent epidermis (Figure 1B). No chd4a signal was detected in uninjured fins or during early stages of regeneration (8 and 24 hpa) (see Additional file 1: Figure S3). chd 4 a expression was initially weak, starting at 2 dpa during blastema formation (see Additional file 1: Figure S3). A robust signal was observed at $3 \mathrm{dpa}$, and then the expression persisted in the blastema of regenerating fins during regenerative outgrowth. By contrast, no signals were detected for the two other Mi-2 orthologs, chd4b and chd3, in the regenerating tissue (Figure 1C,D). In embryos, all three
Mi-2 orthologs were expressed with slightly different expression patterns, suggesting that they have different functions during development (see Additional file 1: Figure S4).

Early zebrafish larvae are also able to regenerate their caudal fin folds after amputation with a similar mechanism to that of regenerating adult caudal fins $[43,44]$. Interestingly, chd $4 a$, but neither $c h d 4 b$ nor $c h d 3$, was expressed in the mesenchymal cells of regenerating larval fin folds at $1 \mathrm{dpa}$ (Figure 1E-G). Expression of chd4a mRNA is specific for regenerating fins, as it was not detected in uncut fin folds at the same developmental stage (3 days 
post-fertilization) (data not shown). Altogether, these results show that one of the three Mi-2 orthologs, chd4a, is transcriptionally induced in the blastema of regenerating adult and embryonic fins.

\section{Specific NuRD component orthologs are expressed in the blastema of regenerating fins}

We then investigated whether other NuRD components are also expressed during fin regeneration. The genome of zebrafish encodes orthologs for all components of the vertebrate NuRD complex. BLAST searches identified three MTA orthologs, LOC794477 (mta1) (Gene ID: 794477), mta2 (Gene ID: 326078), and mta3 (Gene ID: 100003254); two RBBP4/7 orthologs, rbb4 (Gene ID: 321726) and rbb4l (Gene ID: 322129); one MBD2 ortholog, $m b d 2$ (Gene ID: 337105); and two MBD3 orthologs, $m b d 3 a$ (Gene ID: 337133) and $m b d 3 b$ (Gene ID: 321217); but only one HDAC1/2 ortholog, hdac1 (Gene ID: 192302). We examined the expression profile of these genes to test whether NuRD components other than chd4a were also specifically expressed in adult regenerating fins. qRT-PCR analysis revealed that transcripts of hdac1, the two RBBP4/7 orthologs $r b b 4$ and $r b b 4 l$, and one of the three MTA orthologs, mta2, were significantly upregulated in adult regenerating fins at 3 dpa compared with 0 hpa, whereas no upregulation was observed for the other orthologs (Figure 2A).

qRT-PCR data were confirmed by ISH on cryosections of adult caudal fins at $3 \mathrm{dpa}$. A single RNA antisense probe was designed for the two RBBP4/7 orthologs $r b b 4$ and $r b b 4 l$ because of their high RNA (75\%) and amino acid (94\%) sequence similarity. Positive signals for hdac1, rbb4, and $m t a 2$ transcripts were detected in the blastema of adult regenerating fins, with an expression pattern similar to that of chd4a (Figure 2B-D). No signals were detected for the orthologs whose expression was not upregulated by qRT-PCR (data not shown). Furthermore, hdac1, rbb4, and mta2 transcripts were also expressed in mesenchymal cells of regenerating larval fin folds at $1 \mathrm{dpa}$ (Figure 2E-G). Thus, the overlapping expression pattern of some NuRD orthologs in fin regenerates raises the possibility that the expression of a specialized NuRD complex composed of Chd4a, $\mathrm{Rbb} 4 / \mathrm{Rbb} 4 \mathrm{l}, \mathrm{Hdac} 1$, and Mta2 is specifically induced in the blastema during fin regeneration.

\section{Morpholino-mediated knockdown of chd4a, mta2, and the two RBBP4 orthologs rbb4 and $r b b 4 l$ impairs fin regeneration} To determine whether these putative NuRD components play a role in fin regeneration, expression of chd4a, mta2, and the two RBBP4 orthologs $r b b 4$ and $r b b 4 l$ was knocked down using vivo-morpholinos (MOs). For chd4a, two different sets of antisense vivo-morpholinos were designed: a translational blocking MO (MOTL) and a splice blocking MO (MOSP). The efficacy of the splice blocking chd4a
MOSP was tested in zebrafish embryos, and was found to specifically impair the splicing of chd4a transcript (see Additional file 1: Figure S5). MOs were injected into the dorsal half of adult regenerating fins at $3 \mathrm{dpa}$, and the uninjected ventral half was used as an internal control. The effects of the MOs were analyzed at 24 hours post-injection (hpi) by comparing the regenerative surfaces of the injected and uninjected fin halves. No significant differences in regeneration were observed in fin regenerate areas injected with the control MO compared with the uninjected areas (Figure 3A,F). However, injection of the translational (MOTL) or the splice (MOSP) blocking chd4a MOs resulted in a significant reduction in regenerative outgrowth compared with the uninjected region or with fin halves injected with the control MO (Figure 3B,C,F). Interestingly, injection of MOs specific for the metastasis-associated gene $m$ ta2 (Figure 3D) or for the two retinoblastoma-binding orthologs $r b b 4$ and rbb4l (Figure 3E) also significantly decreased regenerative outgrowth compared with the uninjected fin halves (Figure 3F). Thus, morpholino-mediated knockdown of the $\mathrm{NuRD}$ components chd4a, mta2, and the two rbb4 orthologs resulted in a significant reduction in regenerative outgrowth in adult caudal fins, suggesting an important role for these epigenetic factors during fin regeneration.

\section{Specific HDAC1 inhibition affects regenerative outgrowth}

To investigate the function of the histone deacetylase Hdac1 during fin regeneration, we used a pharmacological approach to target its activity. Hdac1 is the only HDAC1/2 ortholog encoded by the genome of zebrafish, and is required for development of the retina, the neural crest, and the central nervous system [45-50]. In humans, HDAC1 and HDAC2 can be selectively inactivated with MGCD0103 (Mocetinostat), a class I-specific HDAC inhibitor [51,52]. Sequence alignment revealed that the catalytic domain of zebrafish Hdac1 is highly conserved (93\% identity with the catalytic domain of human HDAC1 and HDAC2), suggesting that MGCD0103 might also be functional in zebrafish (see Additional file 1: Figure S6). In contrast to morpholinos, which have to be injected into the regenerating tissue, chemical inhibitors can be added directly into the fish water. To inhibit Hdac1 activity during fin regeneration, fish were treated with $5 \mu \mathrm{M}$ MGCD0103 for 10 days after fin amputation, or with $0.05 \%$ DMSO as control. The specificity of MGCD0103 treatment was evaluated by measuring the global acetylation levels of histones $\mathrm{H} 3$ and $\mathrm{H} 4$ in fin regenerates by western blot analysis. We found that the levels of acetylated histones $\mathrm{H} 3$ and $\mathrm{H} 4$ were significantly increased in fin regenerates treated with $5 \mu \mathrm{M}$ MGCD0103 for 4 days compared with fins treated with DMSO (Figure 4A), demonstrating that MGCD0103 effectively blocks Hdac1 activity in the caudal fin during regeneration. Furthermore, no alteration in general 

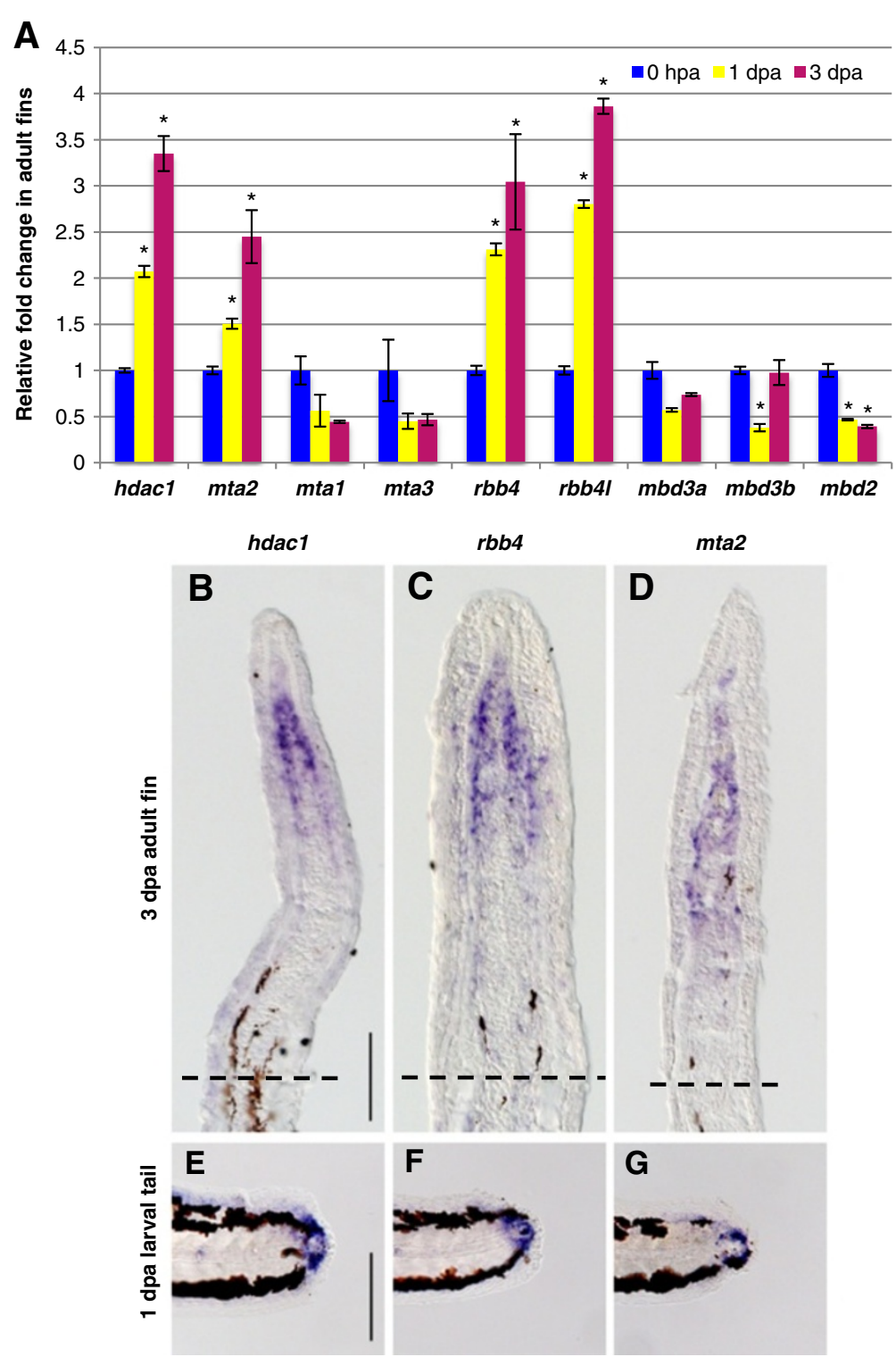

Figure 2 Specific NuRD component orthologs are expressed in the blastema of regenerating fins. (A) Quantitative real-time-PCR analyses of hdac1, mta1, mta2, mta3, rbb4, rbb41, mbd3a, mbd3b, and mbd2 mRNA in regenerating adult caudal fins at 1 and 3 dpa relative to control fins at 0 hpa. Error bars represent the SEM. ${ }^{*} P<0.001$ (B-D) In situ hybridization with hdacl (B), rbb4 (C), and mta2 (D), antisense probes on cryosections of regenerating adult caudal fins at $3 \mathrm{dpa}$. Dashed lines indicate the amputation plane. (E-G) Whole-mount in situ hybridization with hdacl (E), rbb4 (F) and mta2 (G) antisense probes in 1 dpa larval tails. Scale bar: $100 \mu \mathrm{m}$.

health was observed in fish incubated in MGCD0103treated water for 10 days compared with animals incubated with DMSO-treated water (see Additional file 1: Figure S7).

Interestingly, treatment of regenerating fins with $5 \mu \mathrm{M}$ MGCD0103 for 10 days resulted in a substantial reduction in regenerative growth (Figure 4B-E). However, the early stages of the regeneration process seemed not to be affected because wound healing was properly completed and a seemingly normal blastema was formed (Figure 4B,C), suggesting that Hdac1 activity is not essential for the earliest phases of regeneration. Regenerative outgrowth was impaired, starting from $3 \mathrm{dpa}$, and the regeneration process was progressively blocked and finally stopped (Figure 4D,E). Indeed, MGCD0103 treatment for 10 days resulted in the formation of abnormal curled fin-like structures, suggesting differentiation defects. To test whether Hdac1 inhibition also affects fin regeneration after blastema formation, fish were treated with MGCD0103 for 4 days starting at $3 \mathrm{dpa}$. As expected, we found that regenerative growth was blocked, similar to fins that were continuously 


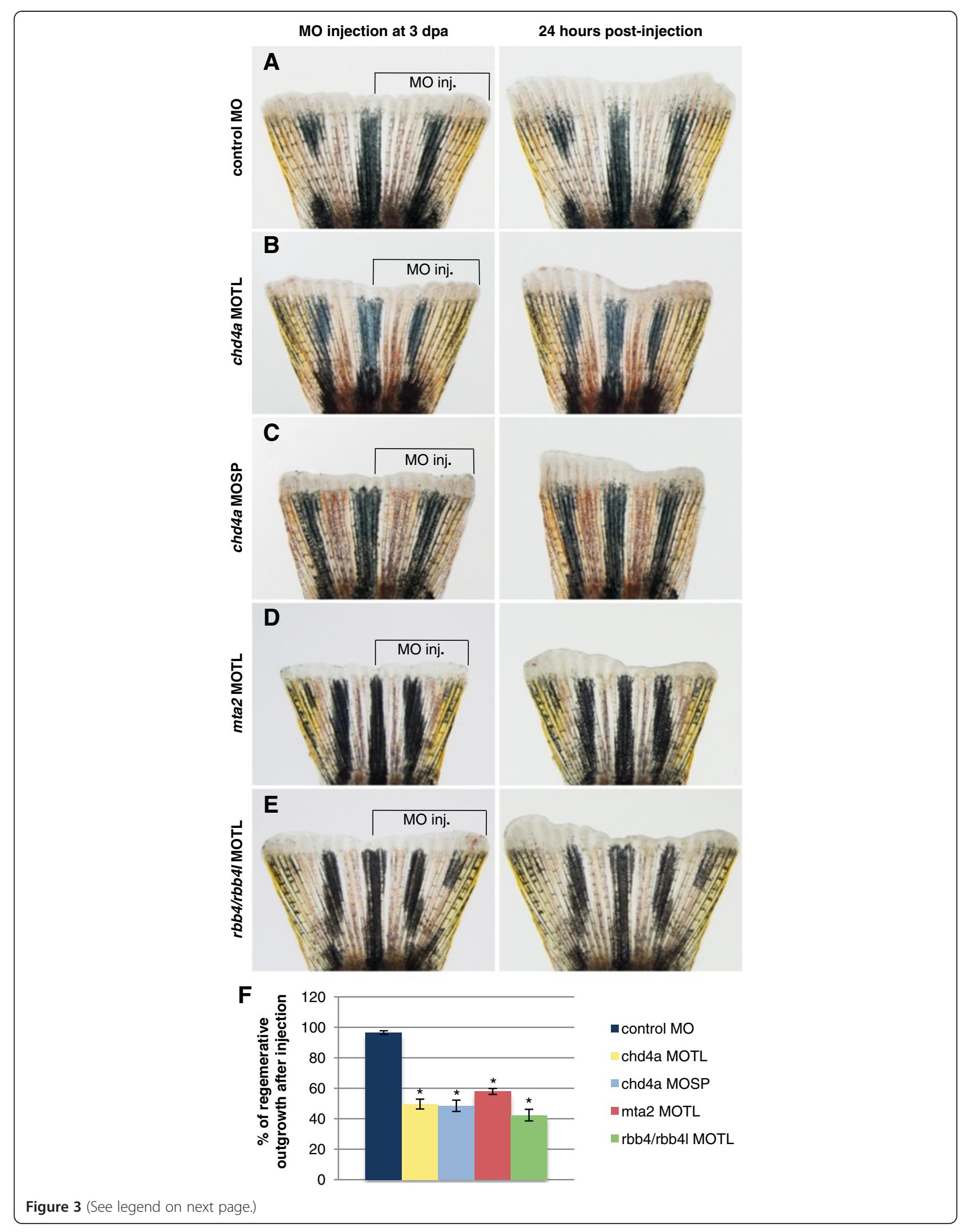


(See figure on previous page.)

Figure 3 Morpholino-mediated knockdown of chd4a, mta2, and the two RBBP4 orthologs $r b b 4$ and rbb4l results in the reduction in regenerative outgrowth. (A-E) The dorsal half of fins was injected with control (A), chd4a translational blocking (B), chd4a splice blocking (C), $m$ ta2 translational blocking (D), and rbb4 + rbb4/ translational blocking (E) vivo-morpholinos (MOs). The uninjected ventral half was used as an internal control to assess normal outgrowth. The left panels show fins shortly after injection at $3 \mathrm{dpa}$ and the right panels show the same fins at 24 hours post-injection (hpi). The control $\mathrm{MO}$ does not affect regenerative outgrowth compared with the uninjected ventral side of the fins. Injection of the antisense MOs specific for chd4a, $m t a 2$, or the two RBBP4 orthologs rbb4 and rbb4/ resulted in reduction in regenerative outgrowth compared with the uninjected side. (F) Percentage of regenerative outgrowth after $\mathrm{MO}$ injection relative to the uninjected control side of the fins. Error bars represent the SEM. $\mathrm{n}=10$. ${ }^{*} P<0.01$.

treated from the time of amputation (see Additional file 1: Figure S8). This result confirmed that Hdac1 inhibition affects regeneration from the onset of regenerative outgrowth.

To test whether MGCD0103 treatment is reversible, fish were exposed to MGCD0103 for 10 days from the time of amputation, and then transferred to normal water for 10 additional days. In general, fins failed to restart the initially blocked regenerative process properly, indicating that the effects of Hdac1 inhibition on caudal fin regeneration are irreversible (see Additional file 1: Figure S9). However,

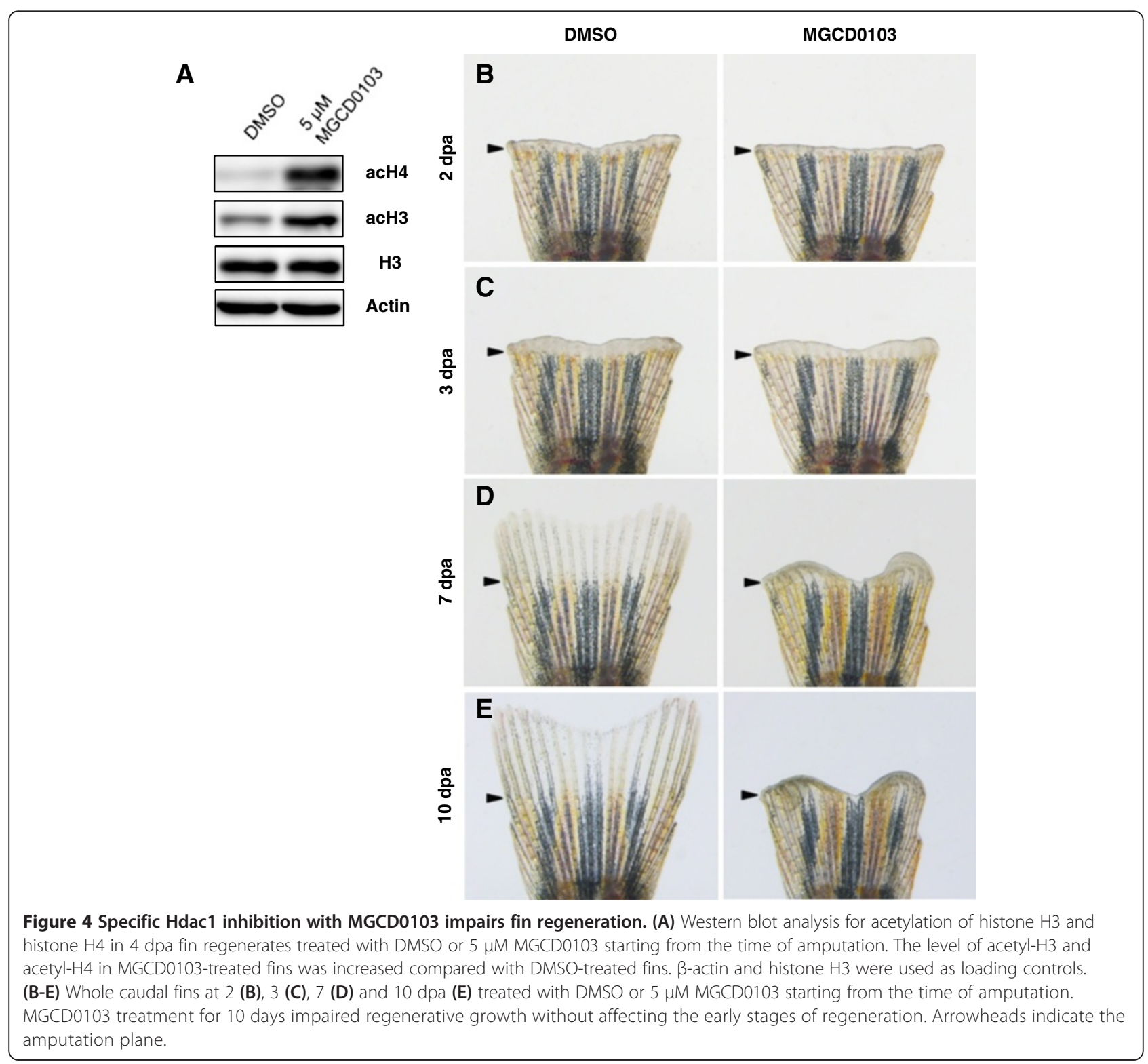


occasionally, a few rays resumed regrowth (see Additional file 1: Figure S9), suggesting that some residual blastema cells retained their original regenerative potential despite the prolonged inhibition of regeneration.

Taken together, these data indicate that MGCD0103mediated inhibition of Hdac1 does not affect wound healing and initial blastema formation, but impairs progression of fin regeneration during the regenerative outgrowth phase.

\section{The NuRD components hdac1, chd $4 a$, mta2, and rbb4 are required for blastema cell proliferation during the regenerative outgrowth phase}

To understand the cause of the regenerative failure in fins deficient in these putative NuRD components, cell proliferation was assessed by labeling DNA-replicating cells with bromodeoxyuridine (BrdU) for 6 hours before fin collection. Because MGCD0103 treatment has the advantage of inhibiting Hdac1 from the time of amputation, cell proliferation was assessed in MGCD0103-treated fin regenerates during blastema formation and during regenerative outgrowth. At 2 dpa, mesenchymal cell proliferation was similar in DMSO-treated and in MGCD0103-treated fins, confirming that Hdac1 does not regulate blastema cell proliferation at this stage (Figure 5A,B,I). However, at 4 $\mathrm{dpa}$, the percentage of BrdU-positive cells was significantly reduced in mesenchymal cells of MGCD0103-treated fish (Figure 5C,D,J). Consistently, cell proliferation was also significantly reduced in the blastema of fin regenerates injected with $c h d 4 a, m t a 2$, or $r b b 4 / r b b 4 l$ MOs at $4 \mathrm{dpa}$, that is, 24 hpi (Figure 5E-H,K). To determine whether the regenerative block was caused by cell death, activation of caspase- 3 was examined by immunostaining to identify apoptotic cells. However, we did not observe any obvious increase in apoptosis in MGCD0103-treated or chd4, $m t a 2$, or $r b b 4 / r b b 4 l$ MO-injected fin regenerates at $4 \mathrm{dpa}$ (see Additional file 1: Figure S10; also data not shown). Altogether, these data suggest that inhibition of Hdac1 and morpholino-mediated knockdown of chd $4 a, m t a 2$, and the two rbb4 orthologs impair fin regeneration by reducing blastema cell proliferation during regenerative outgrowth, without inducing cell death.

MGCD0103 treatment resulted in a noticeable increase in wound epidermis (Figure 5B,D). However, no increase in cell proliferation was detected in the epidermis of MGCD0103-treated fins (Figure 5I,J). MGCD0103 treatment did not alter expression of the wound epidermis markers $w n t 5 b$ and lef1, indicating that hdac1 is not required for the correct specification of the wound epidermis (see Additional file 1: Figure S11). The enlargement of the epidermis in MGCD0103 regenerates could be the result of an abnormal migration of epithelial cells from the stump. As this phenotype was not observed in MO-injected fin regenerates, it is possible that Hdac1 plays an additional role independent of the $\mathrm{Mi}-2 / \mathrm{NuRD}$ complex during fin regeneration.

\section{Depletion of the NuRD components hdac1, chd4a, mta2, and $r b b 4$ results in abnormal patterning of actinotrichia during regeneration}

To examine the cellular consequences of NuRD component depletion, we assessed different cellular markers involved in fin regeneration. First, we examined mesenchymal reorganization by immunostaining with antibodies against Tenascin $\mathrm{C}$, an extracellular matrix glycoprotein. Upon amputation, Tenascin $\mathrm{C}$ is rapidly induced in the mesenchyme below the amputation plane, and then expressed in the regenerating blastema $[16,18]$. We found that Tenascin $C$ expression was normal in both MGCD0103-treated (Figure 6A,B) and chd4a MO-injected fins (see Additional file 1: Figure S12), suggesting that the hdac1 and chd4a do not influence mesenchymal remodeling during blastema formation.

To evaluate the molecular specification of the blastema in fin regenerates deficient in NuRD components, we analyzed the expression of $m s x b$ by ISH. $m s x b$ is a molecular marker of the distal blastema and is required for blastema cell proliferation during fin regeneration [9]. We found that $m s x b$ transcripts were correctly expressed in MGCD0103-treated and in chd4a MO-injected fin regenerates (see Additional file 1: Figure S11), indicating that the distal blastema is correctly specified.

Finally, we analyzed the expression of Actinodin 1, a marker for actinotrichia-forming cells [53]. Actinotrichia are non-mineralized structural components that mechanically support the larval fin fold and the blastema of the fin regenerate [54,55]. The expression pattern of Actinodin 1 was completely disorganized at $4 \mathrm{dpa}$ in fin regenerates treated with MGCD0103, compared with control fins (Figure 6C,D), indicating an abnormal patterning of actinotrichial fibers. A similarly disorganized expression pattern of Actinodin 1 was also observed in fins deficient in chd4a, $m t a 2$, or the two rbb4 orthologs (Figure 6E-H). Altogether, these data suggest that depletion of the NuRD components results in cellular defects after the onset of regenerative outgrowth. Thus, these epigenetic factors are not essential for mesenchymal reorganization or initial blastema formation, but they are required for growth and correct patterning of the blastema during regenerative outgrowth.

\section{Hdac1 inhibition impairs osteoblast differentiation}

To further investigate the effect of Hdac1 inhibition during regeneration of the bony rays, we examined the progression of osteoblast differentiation during regenerative outgrowth. Osteoblasts are specialized cells that line the bony rays and secrete bone matrix. Upon fin amputation, mature osteoblasts dedifferentiate, re-enter the cell cycle, migrate distally in the blastema, and, during regenerative 

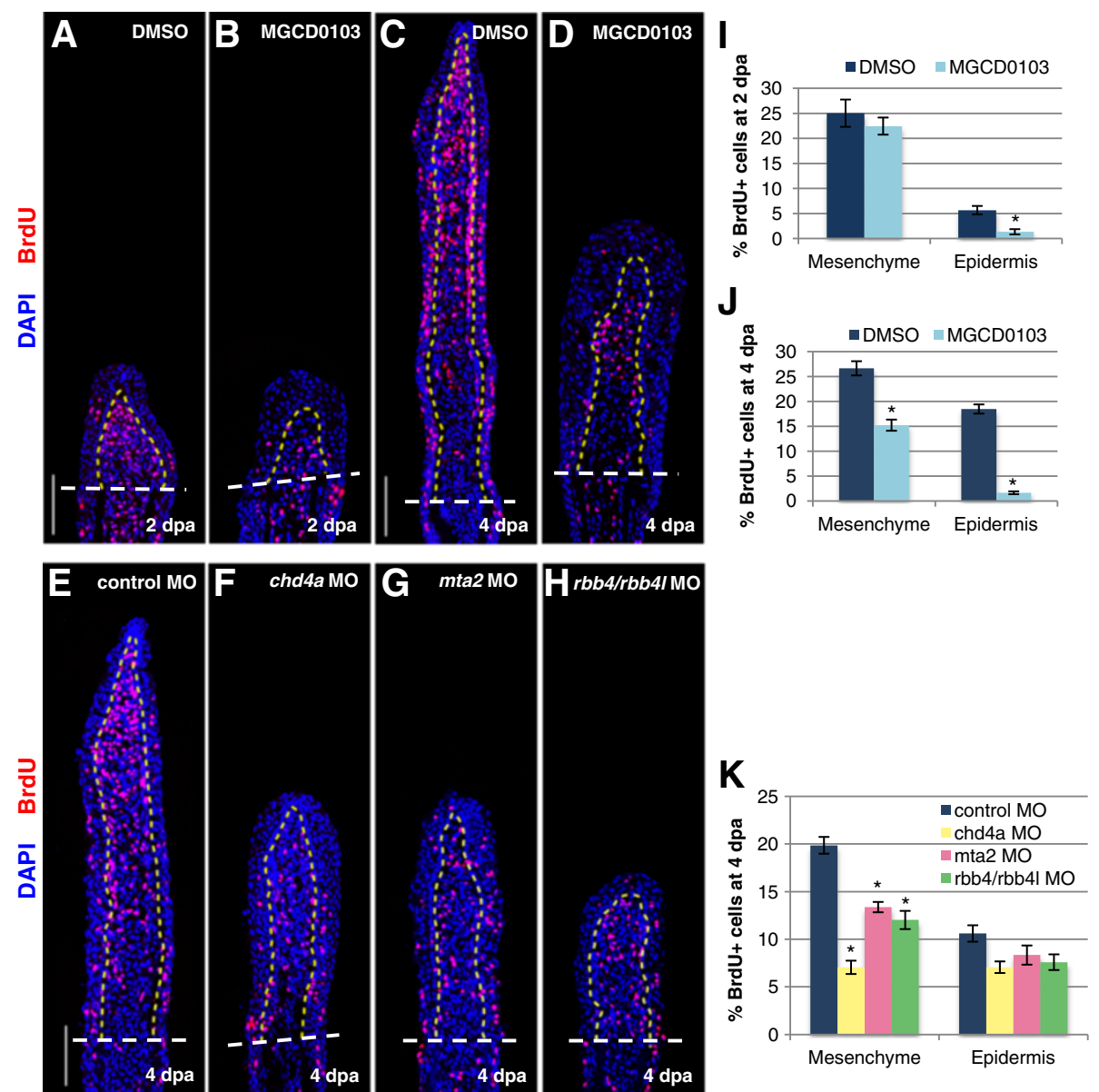

Figure 5 The NuRD components hdac1, chd $4 a, m t a 2$, and $r b b 4$ are required for blastema cell proliferation during the regenerative outgrowth phase. (A-D) Longitudinal sections of fin regenerates treated with DMSO or MGCD0103 at 2 (A-B) or 4 dpa (C-D) stained with BrdU antibody (red) and DAPI (blue). (E-H) Longitudinal sections of fin regenerates at 4 dpa injected with control (E), chd4a (F), mta2 (G), or rbb4+ rbb4l (H) MOs stained with BrdU antibody (red) and DAPI (blue). Yellow dashed lines indicate the boundary between the blastema and the wound epidermis, and white dashed lines indicate the amputation plane. Scale bars: $100 \mu \mathrm{m}$. (I-K) Percentage of BrdU-positive cells relative to the total number of cells (DAPI-labeled) in fin regenerates treated with DMSO or MGCD0103 at $2 \mathrm{dpa}$ (I) or $4 \mathrm{dpa}$ (J) or in fin regenerates injected with control, chd4a, mta2, or rbb4 + rbb4/ MOs (K). Error bars represent the SEM. $n=9 .{ }^{*} P<0.01$.

outgrowth, redifferentiate into osteoblasts in lateral regions of the blastema $[12,56]$. To assess osteoblast proliferation, osteoblasts were double-labeled at 4 dpa with $\mathrm{BrdU}$ and with Zns5, an antibody that marks osteoblasts at all stages of differentiation [57]. In control fins, BrdU-positive osteoblasts can be detected laterally in longitudinal fin sections (Figure 7A). Whereas nuclei of distally located proliferating osteoblasts are characterized by a spherical shape, proximal osteoblast nuclei begin to adopt an elongated shape, characteristic of their differentiated morphology. Interestingly, treatment of fins with $5 \mu \mathrm{M}$ MGCD0103 resulted in a significant reduction in osteoblast proliferation, and the osteoblast nuclei rarely adopted an elongated shape (Figure 7A-C). Similar results were observed in chd4a $\mathrm{MO}$-injected regenerating fins (see Additional file 1: Figure S13). Thus, the histone deacetylase Hdac1 is required for osteoblast proliferation and differentiation during regeneration, and the chromatin-remodeling protein Chd4a also seems to be involved in this process.

Next we used transgenic fish lines expressing fluorescent proteins to examine the expression of the bone differentiation markers runx2, osterix, and osteocalcin, which are sequentially activated during osteoblast differentiation $[10,12]$. In control fish, expression of the preosteoblast marker runx 2 and the intermediate osteoblast marker osterix is relatively low in unamputated fins, and it becomes strongly activated in the blastema during fin regeneration [12] (Figure 7D,F). In MGCD0103-treated fins, runx2:GFP and osterix:mCherry were both reactivated normally in the blastema at $3 \mathrm{dpa}$ (Figure 7D-G), indicating that osteoblast dedifferentiation was not affected by Hdac1 

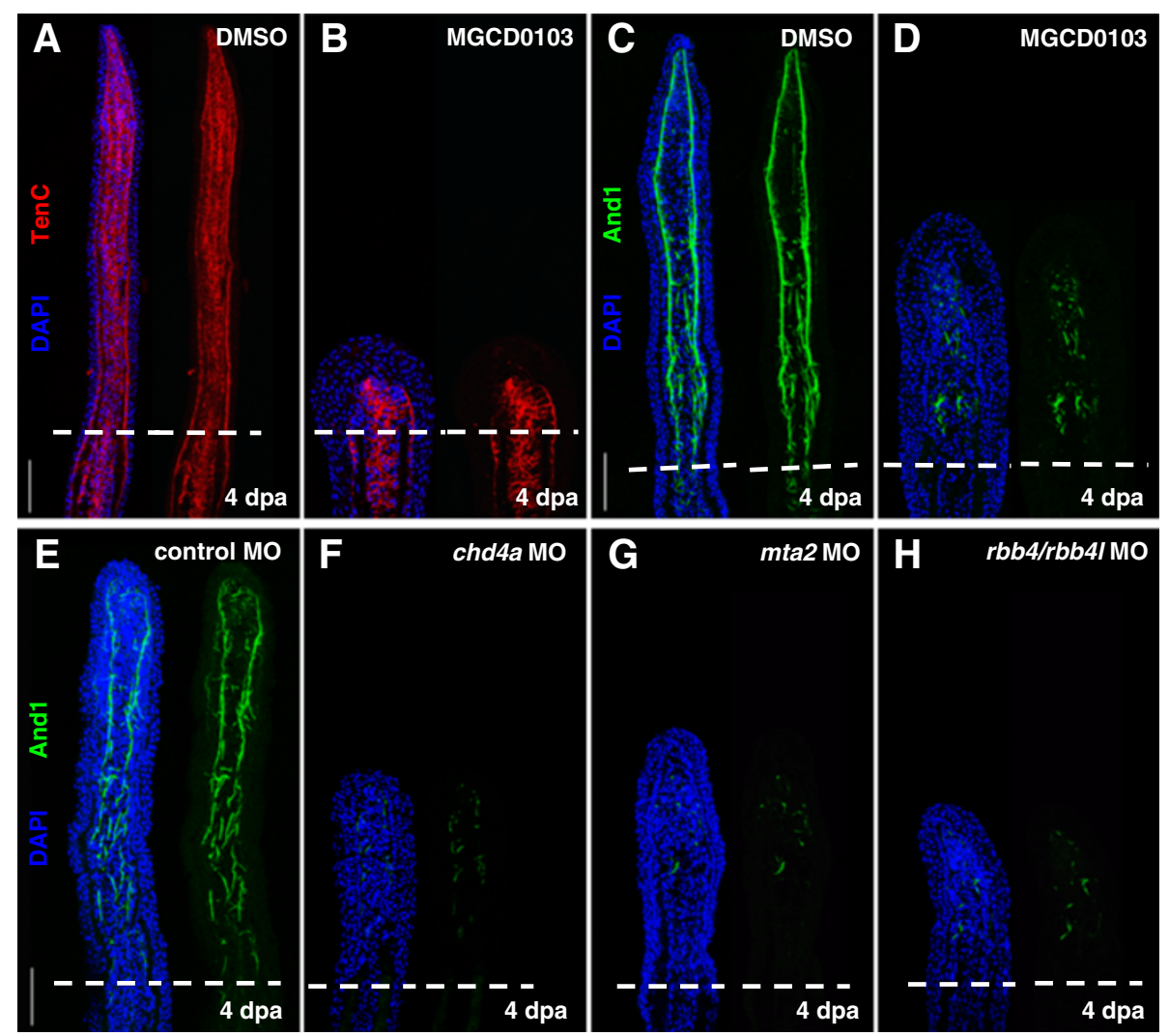

Figure 6 Hdac1 inhibition and morpholino-mediated knockdown of chd4a, mta2, and the two rbb4 orthologs cause abnormal expression of Actinodin 1. (A-B) Longitudinal sections of fin regenerates at 4 dpa treated with DMSO (A) or MGCD0103 (B) and stained with Tenascin C antibody (red) and DAPI (blue). Mesenchymal remodeling was not altered in MGCD0103-treated fins. (C-H) Longitudinal sections of fin regenerates at 4 dpa treated with DMSO (C) or MGCD0103 (D), or injected with control (E), chd4a (F), mta2 (G), or rbb4 + rbb4l (H) MOs stained with Actinodin 1 antibody (green) and DAPI (blue). Depletion of the NuRD components hdac1, chd4a, mta2, or rbb4/rbb4l resulted in a disorganized expression pattern of Actinodin 1. Dashed lines indicate the amputation plane. Scale bars: $100 \mu \mathrm{m}$.

inhibition. However, expression of runx 2 and osterix persisted in the proximal zone at $7 \mathrm{dpa}$, whereas it was progressively downregulated in the proximal differentiating zone in control fins (Figure 7D-G). This indicates a delay in the redifferentiation process in MGCD0103-treated fins.

The late bone differentiation marker osteocalcin, which labels mature osteoblasts, is downregulated in the stump of amputated fins and then robustly re-expressed in the proximal differentiated regenerate [12]. Interestingly, osteocalcin:GFP expression was not reactivated in fin regenerates treated with MGCD0103 at 7 dpa (Figure 7H-I). Furthermore, osteocalcin:GFP expression was also strongly reduced in the blastema of regenerating fins treated with MGCD0103, starting at $3 \mathrm{dpa}$, demonstrating that inhibiting Hdac1 after the blastema has been formed also blocks osteocalcin reactivation (see Additional file 1: Figure S14A). In uninjured fins, MGCD0103 treatment did not alter the expression of osteocalcin:GFP in mature bones (see Additional file 1: Figure S14B), indicating that Hdac1 inhibition specifically blocks the reactivation of osteocalcin:
GFP expression in the differentiating blastema during fin regeneration. Taken together, our results indicate that Hdac1 inhibition prevents redifferentiation of osteoblast precursor cells. However, Hdac1 is not required for osteoblast dedifferentiation following fin amputation.

Hdac1 inhibition results in the upregulation of regeneration marker and two pluripotency-associated genes

In mammalian embryonic stem cells, the NuRD components HDAC1 and MBD3 have previously been shown to directly bind to and control the expression levels of pluripotency-associated factors [58,59]. Therefore, to determine whether Hdac1 also regulates expression of pluripotency-associated factors during regeneration, we measured the expression levels of several candidate genes by qRT-PCR following MGCD0103 treatment. We found that two pluripotency-associated genes, myca and $k l f 4$, were upregulated in MGCD0103-treated fin regenerates at $4 \mathrm{dpa}$ (Figure 8 ). In addition, we found that MGCD0103 treatment also increased the expression 


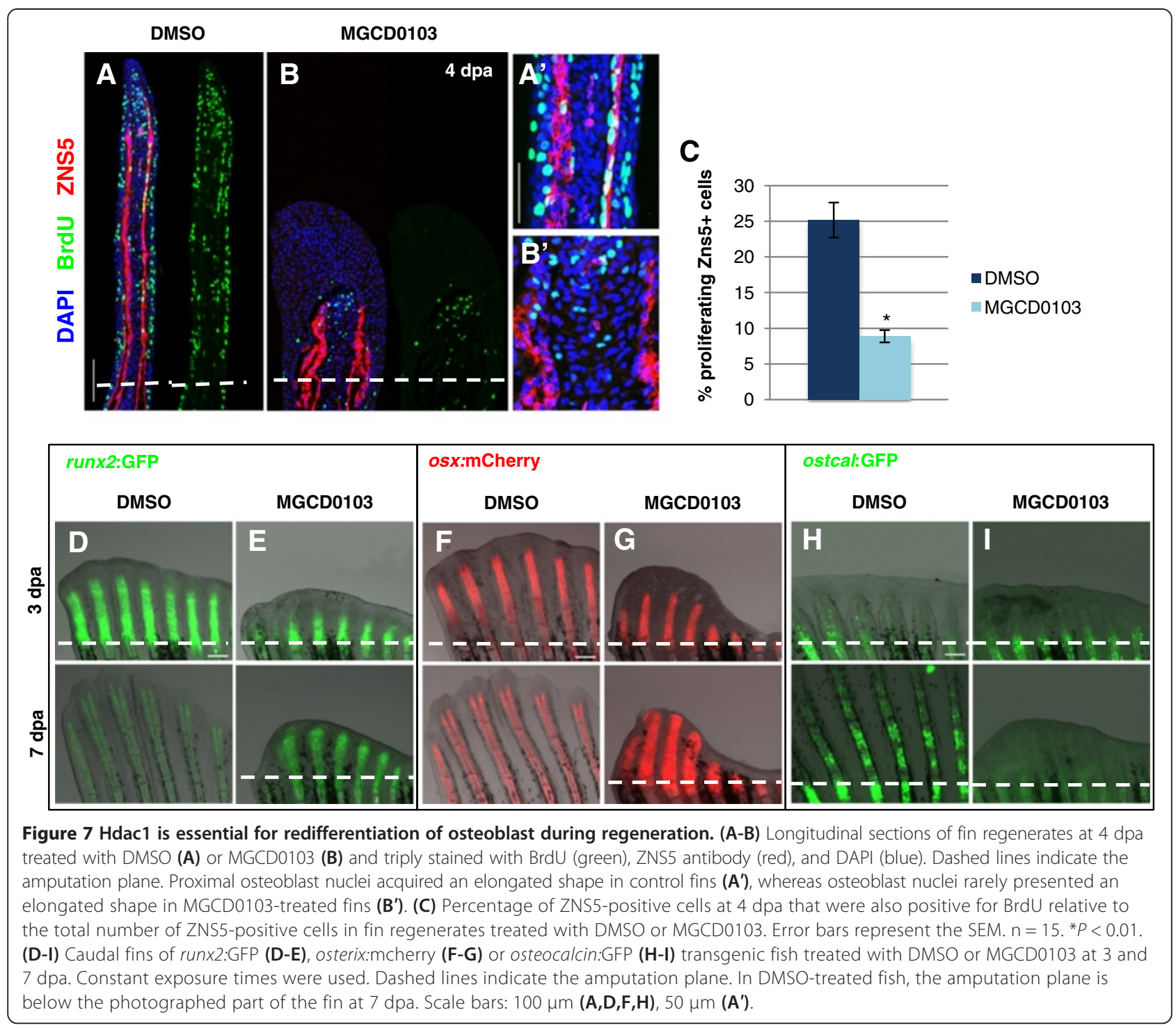

levels of four genes involved in regeneration. junba encodes a transcription factor of the Junb family, which is immediately induced upon fin amputation and required for blastemal proliferation in zebrafish $[44,60]$. The two cathepsins $c t s b a$ and $c t s d$ are proteases whose expression is upregulated during dedifferentiation in regenerating tissues [61,62]. cebpb encodes a bZIP transcription factor upregulated in regenerating liver and required for the proliferative response [63]. Thus, these data demonstrate that Hdac1 represses, directly or indirectly, the transcription of two factors associated with pluripotency, and of several regeneration markers associated with dedifferentiation during regenerative outgrowth.

\section{Discussion}

Here we show evidence for the role of putative NuRD components during fin regeneration in zebrafish. We propose a model in which a specialized $\mathrm{Mi}-2 / \mathrm{NuRD}$ complex could be involved in blastema cell proliferation and redifferentiation during regenerative outgrowth. The zebrafish genome encodes orthologs for every subunit of the vertebrate NuRD complex. However, we found that transcripts of the putative NuRD components $c h d 4 a / \mathrm{Mi}-2$, hdac1/HDAC1/2, rbb4/RBB4/7, and $m t a 2 / M T A$ were specifically co-induced in the blastema during adult and embryonic fin regeneration, and displayed similar spatial and temporal expression patterns. Although there are several homologs for each $\mathrm{NuRD}$ component encoded by the genome of zebrafish (with the exception of hdac1), only one of each seems to be present in the putative NuRD complex involved in fin regeneration. Thus, the combinatorial assembly of the different paralogs of each NuRD subunit may define its specific function. 


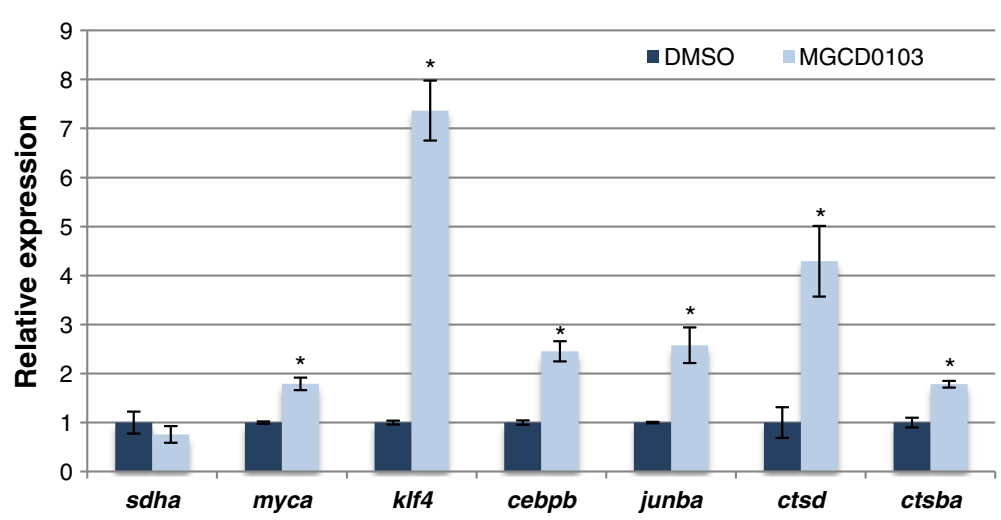

Figure $8 \mathrm{Hdac} 1$ regulates the expression of two pluripotency-associated genes and regeneration marker genes. Quantitative real-timePCR analyses of sdha, myca, klf4, cebpb, junba, ctsd, and ctsba mRNA in MGCD0103-treated fin regenerates relative to DMSO-treated fin regenerates at $4 \mathrm{dpa}$. The relative expression of the housekeeping gene sdha was not changed in MGCD0103-treated fin regenerates, indicating that Hdac1 inhibition does not cause a general increase in gene expression. Error bars represent the SEM. ${ }^{*} P<0.005$.

We also found that disruption of these putative 'regenerating' NuRD components impaired fin regeneration. Chemical inhibition of Hdac1 by MGCD0103 and morpholino-mediated knockdown of chd $4 a, m t a 2$, and the two rbb4 orthologs resulted in the reduction in blastema cell proliferation during regenerative outgrowth. However, these putative NuRD components seem not to be required for the earliest stages of fin regeneration. This is demonstrated by the facts that inhibition of Hdac1 starting from the time of amputation had no influence on wound healing and blastema formation. In addition, Tenascin $C$, an early mesenchymal marker, and $m s x b$, a marker of the distal blastema, were normally expressed in chd4a-deficient and hdac1-deficient fin regenerates.

The wound epidermis was noticeably enlarged in hdac1deficient fin regenerates. It is likely that the increase in the epidermis size resulted from the migration of epithelial cells from the stump, as no increase in cell proliferation was detected in the wound epidermis of MGCD0103-treated fins. Although Hdac1 inhibition reduced cell proliferation in the blastema, epithelial cells might continue to migrate and accumulate, forming an enlarged wound epidermis. This phenotype was not observed in fins deficient in the other NuRD components chd4a, mta2, and rbb4. As HDAC1 is also known to be a catalytic subunit of other multiprotein complexes in mammals, such as CoREST and Sin3 complexes [64], we cannot exclude that Hdac1 plays additional roles independent of the NuRD complex during fin regeneration. Further experiments are needed to identify direct interacting partners of these proteins in regenerating fins.

We found that on addition to the proliferation defects of blastema cells during regenerative outgrowth, Hdac1 inhibition and knockdown of chd4a, mta2, and the two rbb4 orthologs resulted in an abnormal expression pattern of Actinodin 1, a component of structural fibers called actinotrichia. During development, actinotrichia support the fragile fin fold of the larvae. During regeneration, actinotrichia are formed between the epidermis and the blastema prior to lepidotrichia regrowth, and are probably required for shaping the regenerate [53,55]. Consistently, osteoblast proliferation and differentiation were also impaired in hdac1-deficient fin regenerates. Analysis of the bone differentiation markers runx2, osterix, and osteocalcin, which are sequentially expressed during fin regeneration [12], indicated that Hdac1 inhibition did not interfere with osteoblast dedifferentiation. However, expression of the late bone differentiation marker osteocalcin, expressed only in mature bones, was not reactivated in the redifferentiating proximal fin regenerates after Hdac1 inhibition, suggesting that Hdac1 is essential for redifferentiation of osteoblast precursor cells. Indeed, expression of runx 2 and osterix persisted in the proximal blastema of MGCD0103-treated fins, indicating that blastema cells were blocked in an intermediate state.

The effects of morpholino-mediated knockdown of the other NuRD components were not persistent, and regeneration resumed 48 hours post-injection. Morpholino injection has some limitations and is not an appropriate technique to analyze differentiation defects of boneforming cells. Therefore, we were not able to analyze the consequences of morpholino-mediated knockdown of $c h d 4 a, m t a 2$, and $r b b 4$ on osteoblast regeneration.

Somewhat reminiscent to our findings in zebrafish, the planarian ortholog Smed-CHD4 is also essential for regeneration and neoblast differentiation in Schmidtea mediterranea [41]. Smed-CHD4 expression is induced in neoblasts after wounding, and CHD4(RNAi) worms fail to regenerate following amputation or even to maintain normal tissue turnover. In CHD4-depleted animals, the number of neoblast progeny cells is reduced because neoblasts are unable to produce progeny cells committed 
to differentiation [41]. It is, however, not clear whether Smed-CHD4 also acts as a member of a NuRD complex.

Recently, an elegant model has been proposed in which the NuRD complex binds to the promoters of numerous pluripotency genes in embryonic stem cells (ESCs), probably to fine-tune the transcription levels of the genes and to maintain the differentiation responsiveness of the ESCs [59]. In the absence of a functional NuRD complex, expression of these genes is increased above a threshold, thereby blocking the response of ESCs to developmental cues and preventing them from exiting from the self-renewal state [65].

We hypothesize that the Mi-2/NuRD complex might have a similar function during fin regeneration in zebrafish. This is suggested by our findings that the NuRD components were all expressed in the proliferative zone of the blastema during regenerative outgrowth and that their depletion resulted in a reduction in blastema proliferation and an increase in cellular differentiation defects. In addition, Hdac1 inhibition leads to the upregulation of the two pluripotency-associated genes, myca and klf4, and genes encoding regeneration markers associated with dedifferentiation. The histone deacetylase Hdac1 might be required to downregulate the expression of these genes, thereby promoting the responsiveness of blastema cells to regenerative signals in order to ensure correct reconstitution of lost tissues. In the absence of Hdac1, expression of these genes continues to be high, resulting in the blocking of blastema cells in an undifferentiated or partially differentiated state. Further experiments are needed to determine whether Hdac1 represses the expression of these genes in a NuRD-dependent context.

Epigenetic mechanisms are critical for the regulation of gene expression and lineage specification during development [66]. A previous study has shown that H3K27me3 demethylase is required for caudal fin regeneration in zebrafish [67]. Stewart et al. established that many developmental regulatory genes involved in fin regeneration are poised in a bivalent $\mathrm{H} 3 \mathrm{~K} 4 \mathrm{me} 3 / \mathrm{H} 3 \mathrm{~K} 27 \mathrm{me} 3$ chromatin domain, and that the demethylation of H3K27me3 enables activation of expression of these genes in response to injury. It is possible that the zebrafish maintains key developmental regulatory genes in a dormant state to allow rapid switching of their expression profile through epigenetic mechanisms in response to amputation.

\section{Conclusion}

Our study provides further in vivo evidence for the involvement of key epigenetic factors in epimorphic fin regeneration in zebrafish. We propose a model in which a specialized $\mathrm{Mi}-2 / \mathrm{NuRD}$ complex is induced in the blastema of regenerating fins to coordinate proliferation and differentiation and thus reform the missing tissues. Even though different animals may be endowed with different regenerative capacities, crucial regeneration markers are conserved in all vertebrate species. Thus, fin regeneration constitutes an excellent in vivo system to study the epigenetic mechanisms regulating regeneration, and to elucidate how this process is maintained in some vertebrates.

\section{Methods}

The experimental animal research was approved by the cantonal veterinary office of Fribourg (Switzerland).

\section{Zebrafish and fin amputation}

The following zebrafish strains were used in this study: $\mathrm{AB}$ wild-type strain, and the osterix:mCherry (OlSp7: mCherry $^{\text {zf131 }}$ ) [68], runx2:GFP (Has.RUNX2-Mmu.Fos: $\mathrm{EGFP}^{\mathrm{zf} 259}$ ), and osteocalcin:GFP (Ola.Osteocalcin.1:EGF$\mathrm{P}^{\text {hu4008) }}$ fish lines [12]. 6-24 month-old adult fish were anesthetized in $0.1 \%$ tricaine, and the caudal fins were amputated with a razor blade. Animals were allowed to regenerate at $28.5^{\circ} \mathrm{C}$. Larval fin folds were amputated as previously described [43]. Larvae were allowed to regenerate at $28.5^{\circ} \mathrm{C}$ and were collected at $1 \mathrm{dpa}$ for further analysis. For proliferation assays, fish were incubated for 6 hours before fin collection in fish water containing $50 \mu \mathrm{g} / \mathrm{ml}$ BrdU (Sigma-Aldrich, Buchs, Switzerland).

\section{MGCD0103 treatment}

MGCD0103 (Selleckchem, Houston, USA) was dissolved in DMSO at $10 \mathrm{mM}$ stock concentration and then added to the fish water at a final concentration of $5 \mu \mathrm{M}$. $0.05 \%$ DMSO was added to the water of control fish.

\section{Morpholino knockdown}

The following antisense vivo-morpholinos (Gene Tools, OR, USA) were used: chd4a translational blocking (MOTL) 5' -CTCTATCGTCCTCACTGCCGGAC AT-3', chd4a splice blocking (MOSP; targeting the exon 8-intron 8 boundary) 5' -AAAGAGAGTGAGATCCTC ACCCTTT-3', rbb4 MOTL 5' - ACACTTCTTTATCG GCCATTTTGGC-3', rbb4l MOTL 5' - ATGCAGCTTC TTTATCAGCCATAAC-3' and mta2 MOTL 5' - CCGCC ATTCTCTCGCTCTCCTAAAC-3'. The standard vivomorpholino from Gene Tools (5' -CCTCTTACCTCAG TTACAATTTATA-3') was used as negative control. MOs were injected as described previously [16] with a FemtoJet microinjector (Eppendorf) into the dorsal half of regenerating fins at $3 \mathrm{dpa}$. The ventral half of the fins was uninjected, and was used as an internal control. Fins were photographed immediately after injection and at 24 hours postinjection with a stereomicroscope (MZ16) and camera (DFC480) (both Leica). The percentage of regeneration was calculated as previously described $[18,26]$. Areas of the dorsal and the ventral half of regenerating fins were measured 
with Image J 1.43 q software. The percentage of regeneration was calculated with the following formula:

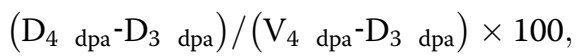

where $\mathrm{D}$ is the area of the dorsal side and $\mathrm{V}$ is the area of the ventral side of the fin regenerate. Statistical significance was determined with the Student's t-test, and significance was set at $P<0.01$.

\section{In situ hybridization}

Whole-mount in situ hybridization and in situ hybridization on fin cryosections was performed as previously described $[22,69]$. Normarski imaging was performed with a Zeiss Axioplan microscope. The following primers were used to generate ISH probes:

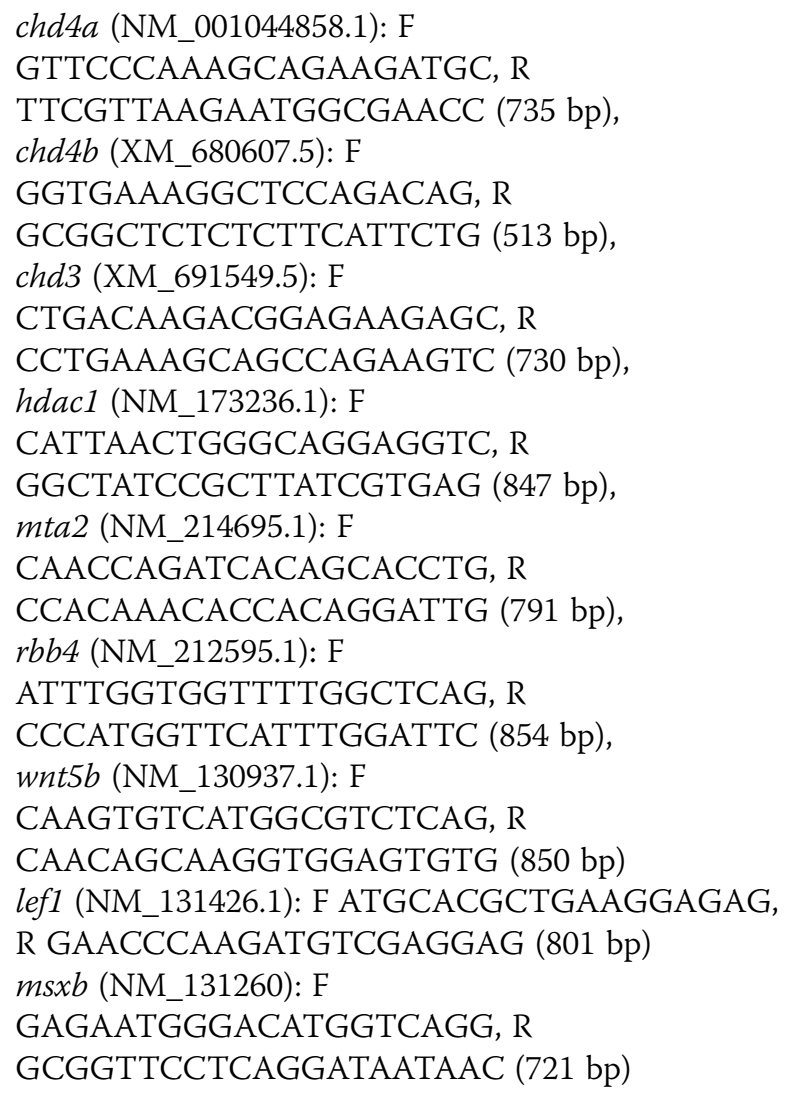

\section{Immunohistochemistry}

Fins were fixed in $4 \%$ paraformaldehyde in PBS, embedded in OCT, and cryosectioned. Antibody staining was performed as previously described [22]. The following primary antibodies were used: rat anti-BrdU (1:200), rabbit anti-active-caspase 3 (1:10000) (both Abcam), rabbit anti-Tenascin C (1:500; US Biological), mouse anti-Zns5 (1:100; Zebrafish International Resource Center), rabbit anti-And1 (1:5000; Eurogentec). The following secondary antibodies were used at a concentration of 1:500: goat anti-rat Alexa Fluor 488 (Molecular Probes), and goat anti-rabbit Cy3-conjugated and anti-mouse Cy5-conjugated antibodies (Jackson ImmunoResearch).

For proliferation assay, BrdU-positive cells distal to the amputation plane were counted in the mesenchyme and epidermis, and the number of BrdU-positive cells was normalized to the total number of DAPI-stained nuclei. Fluorescent pictures were taken with a confocal microscope (TCS SP5; Leica) and Image J 1.43q software was used for the measurements.

\section{Quantitative real-time PCR}

Fin regenerates were collected and total RNA was extracted using Qiazol (Qiagen, Basel, Switzerland). cDNA was synthesized using the QuantiTect Reverse Transcription Kit (Qiagen, Basel, Switzerland). Quantitative real-time PCR was performed in triplicate using the SensiMix SYBR No-ROX Kit (Bioline, Luckenwalde, Germany). Relative expression levels were normalized to $\beta$-actin levels. At least two independent experiments were performed for each target, and data were pooled to generate mean normalized RNA levels. The following primers were used for $\mathrm{qPCR}$ experiments:

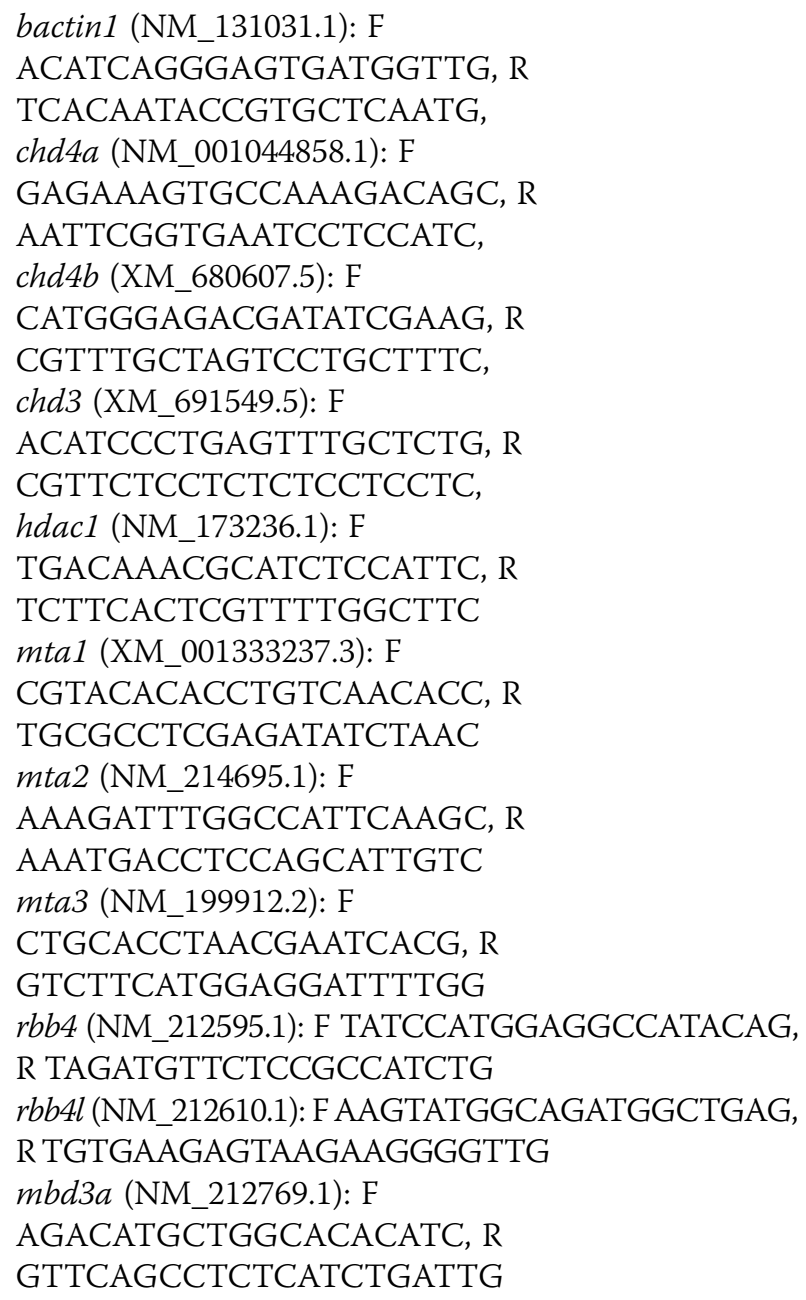


mbd3b (NM_212580.1): F

AGCACAGGTATTTAGATGTGTCTG, R

GCTAATCTGGGAGATGAAAATG

mbd2 (NM_212768.1): F

CTGCAAAGCGTTCAGTGTTAC, R

GCCTGTGGGATCTCTCTAAAC

klf4 (NM_131723.1): F

GACGCACACAGGTGAGAAG, $\mathrm{R}$

GTCCGGTGTGTTTCCTGTAG

myca (NM_131412.1): F

GGCAGCGATTCAGAAGATG, R

CTTTTCTGTCGCTTTTCCAC

cebpb (NM 131884.2): F

GACGCGAGAGGAACAATCTC, $\mathrm{R}$

GCTTCTGTAACCGGTCGTTC

ctsd (NM_131710.1): F

CATCGGCAGTGGACTATCTC, $\mathrm{R}$

CCATGTACTCTCCCTGCATC

ctsba (NM_213336.2): F

TTTGGGAAGACGTCCTACAG, $\mathrm{R}$

AGCAGGAAATCCTCATAGACC

junba (NM_213556.3): F

AGTACCACCACCATCACCAC, R

GTCTGTGGCTCCTCTTTCAG

sdha (NM_200910.1): F

TGTGTGGAACACTGATCTGG, R

TCCACACGATCCTTGAAGTC

For MOSP efficacy, segments of the correctly spliced

chd $4 a$ mRNA around the exon 8 were amplified with

the following primers:

prCP46: F TCCTTATCGTGACAGGCCTAC

prCP47: R GGAGTAGGGCCCTTTCAATC

prCP82: R AAGCAGACCATGTGATAGGC

\section{Western blot}

Fin regenerates were disrupted using glass beads in a mixture of $240 \mathrm{mM}$ Tris $\mathrm{HCl} \mathrm{pH} \mathrm{6.8,} \mathrm{8 \%} \mathrm{SDS,} \mathrm{40 \%} \mathrm{glycerol,}$ $0.01 \%$ bromophenol blue, and $1.4 \mathrm{M} \beta$-mercaptoethanol. Then $20 \mu \mathrm{g}$ of total proteins were loaded per lane and separated by SDS-PAGE (12\%). Even loading was verified by staining with Ponceau $S$ and with $\beta$-actin antibodies (1:2000; Sigma). Proteins were transferred onto nitrocellulose membranes, and blots were incubated in $5 \%$ milk with rabbit anti-Histone $\mathrm{H} 3$ (1:2000), rabbit anti-acetyl-histone H3 (1:1000), anti-acetyl-histone H4 (1:1000) (all Millipore) and $\beta$-actin (1:2000; Sigma). Secondary HRP anti-rabbit and anti-mouse antibodies (Sigma) were used at 1:10,000.

\section{Additional file}

Additional file 1: Figure S1. The genome of zebrafish encodes three $\mathrm{Mi}-2$ orthologs. Figure S2. One of the three Mi-2 homologs, chd4a, is induced during regeneration in the adult caudal fin. Figure S3. chd4a is expressed during regenerative outgrowth in adult caudal fin. Figure S4. chd4a, chd4b, and chd3 are expressed in developing zebrafish embryos. Figure S5. The splice blocking antisense chd4a MOSP efficiently impairs the splicing of chd4a transcript. Figure S6. Zebrafish $\mathrm{Hdac1}$ and human HDAC1/HDAC2 are highly conserved. Figure S7. MGCD0103 treatment does not affect the general health of zebrafish. Figure S8. Hdac1 inhibition after blastema formation is sufficient to impair regenerative outgrowth. Figure S9. The effects of MGCD0103 treatment are not reversible. Figure S10. Hdac1 inhibition and chd4a knockdown do not result in the activation of the apoptosis marker caspase-3. Figure S11. wnt5b, lef1, and $m s \times b$ are expressed in chd4a morpholino oligonucleotide (MO)-injected and MGCD0103-treated fins. Figure S12. chd4a knockdown does not affect Tenascin C expression. Figure S13. chd4a knockdown reduces osteoblast proliferation. Figure $\mathbf{S 1 4}$. Hdac1 inhibition after blastema formation is sufficient to block reactivation of osteocalcin expression.

\section{Abbreviations}

And1: Actinodin 1; BrdU: 5-bromo-2'-deoxyuridine; DAPI: 4',6-diamidino-2phenylindole; DMSO: Dimethyl sulfoxide; dpa: Day post-amputation; hpa: Hour post-amputation; MO: Vivo-morpholino; MOSP: Splice blocking vivo-morpholino; MOTL: Translational blocking vivo-morpholino; qRTPCR: quantitative real-time polymerase chain reaction; TenC: Tenascin C.

\section{Competing interests}

The authors declare no competing interests.

\section{Authors' contributions}

FM, CW, and AJ conceived and planned the study. CP performed the experiments. CP, FM, CW, and AJ wrote the manuscript. All the authors discussed the results and commented on the manuscript. All authors read and approved the final manuscript.

\section{Acknowledgements}

We are grateful to Franscesco Hofmann, Novartis Pharma AG for providing the NPV-AEW541 compounds, to Claire Jacob for providing the MGCD0103 compound, and to Gilbert Weidinger for providing the transgenic fish. We thank V. Zimmerman for excellent fish care; S. Käser-Pébernard for critical reading of the manuscript; and $Y$. Molleyres and $L$. Bulliard for excellent technical help. This work was funded by the Swiss National Funds grants 31003A_125577 of F. M. and C.W. and 310030_138062 of A. J.

Received: 6 November 2013 Accepted: 23 April 2014 Published: 29 April 2014

\section{References}

1. Becker T, Wullimann MF, Becker CG, Bernhardt RR, Schachner M: Axonal regrowth after spinal cord transection in adult zebrafish. J Comp Neurol 1997, 377:577-595

2. Bernhardt RR, Tongiorgi E, Anzini P, Schachner M: Increased expression of specific recognition molecules by retinal ganglion cells and by optic pathway glia accompanies the successful regeneration of retinal axons in adult zebrafish. J Comp Neurol 1996, 376:253-264.

3. Poss KD, Wilson LG, Keating MT: Heart regeneration in zebrafish. Science 2002, 298:2188-2190.

4. Poss KD, Keating MT, Nechiporuk A: Tales of regeneration in zebrafish. Dev Dyn 2003, 226:202-210.

5. Sadler KC, Krahn KN, Gaur NA, Ukomadu C: Liver growth in the embryo and during liver regeneration in zebrafish requires the cell cycle regulator, uhrf1. Proc Natl Acad Sci U S A 2007, 104:1570-1575.

6. Akimenko M-A, Marí-Beffa M, Becerra J, Géraudie J: Old questions, new tools, and some answers to the mystery of fin regeneration. Dev Dyn 2003, 226:190-201.

7. Iovine MK: Conserved mechanisms regulate outgrowth in zebrafish fins. Nat Chem Biol 2007, 3:613-618.

8. Gurley KA, Alvarado AS: Stem cells in animal models of regeneration. StemBook 2008. ed. The Stem Cell Research Community, StemBook, doi/10.3824/stembook.1.32.1.

9. Nechiporuk A, Keating MT: A proliferation gradient between proximal and msxb-expressing distal blastema directs zebrafish fin regeneration. Development 2002, 129:2607-2617. 
10. Brown AM, Fisher S, lovine MK: Osteoblast maturation occurs in overlapping proximal-distal compartments during fin regeneration in zebrafish. Dev Dyn 2009, 238:2922-2928.

11. Lee Y, Hami D, De Val S, Kagermeier-Schenk B, Wills AA, Black BL, Weidinger G, Poss KD: Maintenance of blastemal proliferation by functionally diverse epidermis in regenerating zebrafish fins. Dev Biol 2009, 331:270-280.

12. Knopf F, Hammond C, Chekuru A, Kurth T, Hans S, Weber CW, Mahatma G, Fisher S, Brand M, Schulte-Merker S, Weidinger G: Bone regenerates via dedifferentiation of osteoblasts in the zebrafish fin. Dev Cell 2011, 20:713-724.

13. Kragl M, Knapp D, Nacu E, Khattak S, Maden M, Epperlein HH, Tanaka EM: Cells keep a memory of their tissue origin during axolotl limb regeneration. Nature 2009, 460:60-65.

14. Tu S, Johnson SL: Fate restriction in the growing and regenerating zebrafish fin. Dev Cell 2011, 20:725-732.

15. Singh SP, Holdway JE, Poss KD: Regeneration of amputated zebrafish fin rays from de novo osteoblasts. Dev Cell 2012, 22:879-886.

16. Chablais F, Jazwinska A: IGF signaling between blastema and wound epidermis is required for fin regeneration. Development 2010, 137:871-879.

17. Grotek B, Wehner D, Weidinger G: Notch signaling coordinates cellular proliferation with differentiation during zebrafish fin regeneration. Development 2013, 140:1412-1423.

18. Jaźwińska A, Badakov R, Keating MT: Activin-betaA signaling is required for zebrafish fin regeneration. Curr Biol 2007, 17:1390-1395.

19. Lee Y, Grill S, Sanchez A, Murphy-Ryan M, Poss KD: Fgf signaling instructs position-dependent growth rate during zebrafish fin regeneration. Development 2005, 132:5173-5183.

20. Münch J, González-Rajal A, de la Pompa JL: Notch regulates blastema proliferation and prevents differentiation during adult zebrafish fin regeneration. Development 2013, 140:1402-1411.

21. Poss KD, Shen J, Nechiporuk A, McMahon G, Thisse B, Thisse C, Keating MT: Roles for Fgf signaling during zebrafish fin regeneration. Dev Biol 2000, 222:347-358.

22. Poss KD, Shen J, Keating MT: Induction of lef1 during zebrafish fin regeneration. Dev Dyn 2000, 219:282-286.

23. Smith A, Avaron F, Guay D, Padhi BK, Akimenko MA: Inhibition of BMP signaling during zebrafish fin regeneration disrupts fin growth and scleroblasts differentiation and function. Dev Biol 2006, 299:438-454.

24. Stoick-Cooper CL, Weidinger G, Riehle KJ, Hubbert C, Major MB, Fausto N, Moon RT: Distinct Wnt signaling pathways have opposing roles in appendage regeneration. Development 2007, 134:479-489.

25. Tawk M, Tuil D, Torrente Y, Vriz S, Paulin D: High-efficiency gene transfer into adult fish: a new tool to study fin regeneration. Genesis 2002, 32:27-31.

26. Thummel R, Bai S, Sarras MP Jr, Song P, McDermott J, Brewer J, Perry M, Zhang X, Hyde DR, Godwin AR: Inhibition of zebrafish fin regeneration using in vivo electroporation of morpholinos against fgfr 1 and msxb. Dev Dyn 2006, 235:336-346.

27. White JA, Boffa MB, Jones B, Petkovich M: A zebrafish retinoic acid receptor expressed in the regenerating caudal fin. Development 1994, 120:1861-1872.

28. Whitehead GG, Makino S, Lien C-L, Keating MT: fgf20 is essential for initiating zebrafish fin regeneration. Science 2005, 310:1957-1960.

29. Denslow SA, Wade PA: The human Mi-2/NuRD complex and gene regulation. Oncogene 2007, 26:5433-5438.

30. Tong JK, Hassig CA, Schnitzler GR, Kingston RE, Schreiber SL: Chromatin deacetylation by an ATP-dependent nucleosome remodelling complex. Nature 1998, 395:917-921.

31. Wade PA, Gegonne A, Jones PL, Ballestar E, Aubry F, Wolffe AP: Mi-2 complex couples DNA methylation to chromatin remodelling and histone deacetylation. Nat Genet 1999, 23:62-66.

32. Xue Y, Wong J, Moreno GT, Young MK, Côté J, Wang W: NURD, a novel complex with both ATP-dependent chromatin-remodeling and histone deacetylase activities. Mol Cell 1998, 2:851-861.

33. Zhang Y, LeRoy G, Seelig HP, Lane WS, Reinberg D: The dermatomyositis-specific autoantigen $\mathrm{Mi} 2$ is a component of a complex containing histone deacetylase and nucleosome remodeling activities. Cell 1998, 95:279-289.

34. Allen HF, Wade PA, Kutateladze TG: The NuRD architecture. Cell Mol Life Sci 2013, 70:3513-3524

35. Bowen NJ, Fujita N, Kajita M, Wade PA: Mi-2/NuRD: multiple complexes for many purposes. Biochim Biophys Acta 2004, 1677:52-57.
36. Von Zelewsky T, Palladino F, Brunschwig K, Tobler H, Hajnal A, Müller F: The C. elegans $\mathrm{Mi}-2$ chromatin-remodelling proteins function in vulval cell fate determination. Development 2000, 127:5277-5284.

37. Unhavaithaya Y, Shin TH, Miliaras N, Lee J, Oyama T, Mello CC: MEP-1 and a homolog of the NURD complex component Mi-2 act together to maintain germline-soma distinctions in C. elegans. Cell 2002, 111:991-1002.

38. Kehle J, Beuchle D, Treuheit S, Christen B, Kennison JA, Bienz M, Müller J: $\mathrm{dMi}-2$, a hunchback-interacting protein that functions in polycomb repression. Science 1998, 282:1897-1900.

39. Yoshida T, Hazan I, Zhang J, Ng SY, Naito T, Snippert HJ, Heller EJ, Qi X, Lawton LN, Williams CJ, Georgopoulos K: The role of the chromatin remodeler Mi-2beta in hematopoietic stem cell self-renewal and multilineage differentiation. Genes Dev 2008, 22:1174-1189.

40. Kaji K, Caballero IM, MacLeod R, Nichols J, Wilson VA, Hendrich B: The NuRD component $\mathrm{Mbd} 3$ is required for pluripotency of embryonic stem cells. Nat Cell Biol 2006, 8:285-292.

41. Scimone ML, Meisel J, Reddien PW: The Mi-2-like Smed-CHD4 gene is required for stem cell differentiation in the planarian Schmidtea mediterranea. Development 2010, 137:1231-1241.

42. Zakaria C, Kassahun H, Yang X, Labbé J-C, Nilsen H, Ramotar D: Caenorhabditis elegans APN-1 plays a vital role in maintaining genome stability. DNA Repair (Amst) 2010, 9:169-176.

43. Kawakami A, Fukazawa T, Takeda H: Early fin primordia of zebrafish larvae regenerate by a similar growth control mechanism with adult regeneration. Dev Dyn 2004, 231:693-699.

44. Yoshinari N, Ishida T, Kudo A, Kawakami A: Gene expression and functional analysis of zebrafish larval fin fold regeneration. Dev Biol 2009, 325:71-81.

45. Stadler JA, Shkumatava A, Norton WHJ, Rau MJ, Geisler R, Fischer S, Neumann CJ: Histone deacetylase 1 is required for cell cycle exit and differentiation in the zebrafish retina. Dev Dyn 2005, 233:883-889.

46. Nambiar RM, Ignatius MS, Henion PD: Zebrafish colgate/hdac1 functions in the non-canonical Wnt pathway during axial extension and in Wnt-independent branchiomotor neuron migration. Mech Dev 2007 124:682-698.

47. Ignatius MS, Unal Eroglu A, Malireddy S, Gallagher G, Nambiar RM, Henion PD: Distinct functional and temporal requirements for zebrafish Hdac1 during neural crest-derived craniofacial and peripheral neuron development. PLoS One 2013, 8:e63218.

48. Yamaguchi M, Tonou-Fujimori N, Komori A, Maeda R, Nojima Y, Li H, Okamoto $\mathrm{H}$, Masai l: Histone deacetylase 1 regulates retinal neurogenesis in zebrafish by suppressing Wnt and Notch signaling pathways. Development 2005, 132:3027-3043.

49. Harrison MRM, Georgiou AS, Spaink HP, Cunliffe VT: The epigenetic regulator Histone Deacetylase 1 promotes transcription of a core neurogenic programme in zebrafish embryos. BMC Genomics 2011, 12:24

50. Cunliffe $V T$ : Histone deacetylase 1 is required to repress Notch target gene expression during zebrafish neurogenesis and to maintain the production of motoneurones in response to hedgehog signalling. Development 2004, 131:2983-2995.

51. Fournel M, Bonfils C, Hou Y, Yan PT, Trachy-Bourget M-C, Kalita A, Liu J, Lu A-H, Zhou NZ, Robert M-F, Gillespie J, Wang JJ, Ste-Croix H, Rahil J, Lefebvre S, Moradei O, Delorme D, Macleod AR, Besterman JM, Li Z: MGCD0103, a novel isotype-selective histone deacetylase inhibitor, has broad spectrum antitumor activity in vitro and in vivo. Mol Cancer Ther 2008, 7:759-768.

52. Khan N, Jeffers M, Kumar S, Hackett C, Boldog F, Khramtsov N, Qian X, Mills E, Berghs SC, Carey N, Finn PW, Collins LS, Tumber A, Ritchie JW, Jensen PB, Lichenstein HS, Sehested M: Determination of the class and isoform selectivity of small-molecule histone deacetylase inhibitors. Biochem J 2008, 409:581-589.

53. Zhang J, Wagh P, Guay D, Sanchez-Pulido L, Padhi BK, Korzh V, Andrade-Navarro MA, Akimenko M-A: Loss of fish actinotrichia proteins and the fin-to-limb transition. Nature 2010, 466:234-237.

54. Santamaría JA, Becerra J: Tail fin regeneration in teleosts: cell-extracellular matrix interaction in blastemal differentiation. J Anat 1991, 176:9-21.

55. Durán I, Marí-Beffa M, Santamaría JA, Becerra J, Santos-Ruiz L: Actinotrichia collagens and their role in fin formation. Dev Biol 2011, 354:160-172.

56. Sousa S, Afonso N, Bensimon-Brito A, Fonseca M, Simões M, Leon J, Roehl $\mathrm{H}$, Cancela ML, Jacinto A: Differentiated skeletal cells contribute to blastema formation during zebrafish fin regeneration. Development 2011, 138:3897-3905 
57. Johnson SL, Weston JA: Temperature-sensitive mutations that cause stage-specific defects in Zebrafish fin regeneration. Genetics 1995, 141:1583-1595.

58. Kidder BL, Palmer S: HDAC1 regulates pluripotency and lineage specific transcriptional networks in embryonic and trophoblast stem cells. Nucleic Acids Res 2012, 40:2925-2939.

59. Reynolds N, Latos P, Hynes-Allen A, Loos R, Leaford D, O'Shaughnessy A, Mosaku O, Signolet J, Brennecke P, Kalkan T, Costello I, Humphreys P, Mansfield W, Nakagawa K, Strouboulis J, Behrens A, Bertone P, Hendrich B: NuRD suppresses pluripotency gene expression to promote transcriptional heterogeneity and lineage commitment. Cell Stem Cell 2012, 10:583-594.

60. Ishida T, Nakajima T, Kudo A, Kawakami A: Phosphorylation of Junb family proteins by the Jun $\mathrm{N}$-terminal kinase supports tissue regeneration in zebrafish. Dev Biol 2010, 340:468-479.

61. Ju BG, Kim WS: Upregulation of cathepsin D expression in the dedifferentiating salamander limb regenerates and enhancement of its expression by retinoic acid. Wound Repair Regen 1998, 6:349-357.

62. Mathew LK, Sengupta S, Franzosa JA, Perry J, La Du J, Andreasen EA, Tanguay RL: Comparative expression profiling reveals an essential role for raldh2 in epimorphic regeneration. J Biol Chem 2009, 284:33642-33653.

63. Greenbaum LE, Li W, Cressman DE, Peng Y, Ciliberto G, Poli V, Taub R: CCAAT enhancer- binding protein beta is required for normal hepatocyte proliferation in mice after partial hepatectomy. J Clin Invest 1998, 102:996-1007.

64. Yang X-J, Seto E: The Rpd3/Hda1 family of lysine deacetylases: from bacteria and yeast to mice and men. Nat Rev Mol Cell Biol 2008, 9:206-218.

65. Reynolds N, O'Shaughnessy A, Hendrich B: Transcriptional repressors: multifaceted regulators of gene expression. Development 2013, 140:505-512

66. Barrero MJ, Boué S, Izpisúa Belmonte JC: Epigenetic mechanisms that regulate cell identity. Cell Stem Cell 2010, 7:565-570.

67. Stewart S, Tsun Z-Y, Izpisua Belmonte JC: A histone demethylase is necessary for regeneration in zebrafish. Proc Natl Acad Sci U S A 2009, 106:19889-19894.

68. Spoorendonk KM, Peterson-Maduro J, Renn J, Trowe T, Kranenbarg S, Winkler C, Schulte-Merker S: Retinoic acid and Cyp26b1 are critical regulators of osteogenesis in the axial skeleton. Development 2008, 135:3765-3774.

69. Smith A, Zhang J, Guay D, Quint E, Johnson A, Akimenko MA: Gene expression analysis on sections of zebrafish regenerating fins reveals limitations in the whole-mount in situ hybridization method. Dev Dyn 2008, 237:417-425.

doi:10.1186/1741-7007-12-30

Cite this article as: Pfefferli et al: Specific NuRD components are required for fin regeneration in zebrafish. BMC Biology 2014 12:30.

\section{Submit your next manuscript to BioMed Central and take full advantage of:}

- Convenient online submission

- Thorough peer review

- No space constraints or color figure charges

- Immediate publication on acceptance

- Inclusion in PubMed, CAS, Scopus and Google Scholar

- Research which is freely available for redistribution

Submit your manuscript at www.biomedcentral.com/submit
Ciomed Central 